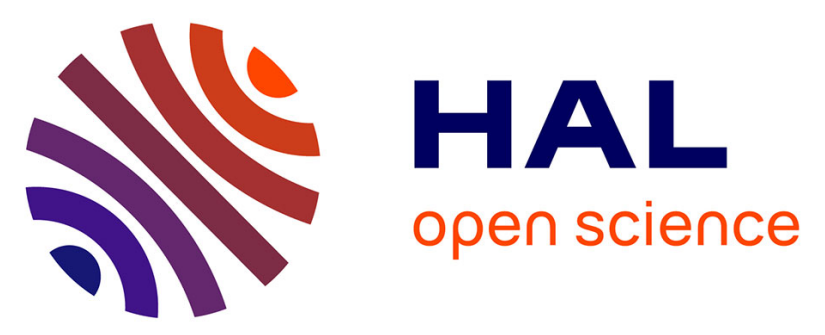

\title{
Simultaneous map-plotting and localization method for unknown driving environment
}

\author{
H. Chou, M. Traonmilin, Edern Ollivier, M. Parent
}

\section{To cite this version:}

H. Chou, M. Traonmilin, Edern Ollivier, M. Parent. Simultaneous map-plotting and localization method for unknown driving environment. IEEE International Conference on Networking, Sensing and Control, 2004, Mar 2004, Taipei, Taiwan. 10.1109/ICNSC.2004.1297064 . hal-01773463

\section{HAL Id: hal-01773463 \\ https://hal.science/hal-01773463}

Submitted on 4 Aug 2018

HAL is a multi-disciplinary open access archive for the deposit and dissemination of scientific research documents, whether they are published or not. The documents may come from teaching and research institutions in France or abroad, or from public or private research centers.
L'archive ouverte pluridisciplinaire HAL, est destinée au dépôt et à la diffusion de documents scientifiques de niveau recherche, publiés ou non, émanant des établissements d'enseignement et de recherche français ou étrangers, des laboratoires publics ou privés. 


\title{
Simultaneous Map-Plotting and Localization Method for Unknown Driving Environment
}

\author{
H. Chou, M. Traonmilin, E. Ollivier, M. Parent \\ Project IMARA, INRIA Rocquencourt \\ B.P. 105, 78153 Le Chesnay Cedex, France \\ hsin.chou@inria.fr
}

\begin{abstract}
Localization is a key issue for automatic navigation of autonomous vehicles. In this article, a simultaneous map-plotting and localization method for an autonomous vehicle is proposed. We use a laser detection and ranging sensor to detect the operating environment. An environment map is plot out using the sensor output data. Then, with an odometer, the vehicle position is located on this map. Finally, the two sensor outputs are merged using a Kalman filter to correct the map as well as the vehicle position.
\end{abstract}

Keywords: Localization, Map-Plotting, Kalman Filter, Autonomous Vehicle.

\section{Introduction}

Automatic navigation of an autonomous vehicle involves following a planned trajectory taking the vehicle from a start configuration to a goal configuration. The desired trajectory can be viewed as a collection of vehicle configurations, which describe the vehicle's location and orientation with respect to the world coordinate system.

One of the major difficulties, however, for this automatic navigation task, is to know the exact location and orientation of the vehicle during its operation. Due to the unavoidable measurement errors, the vehicle configuration is never precisely, if not poorly, determined.

In some previous research work, self positioning systems has been divided into three basic technologies $[3,8]$ : stand alone (e.g. odometer, inertial navigating), satellite-based (e.g. global positioning system), and terrestrial radio-based (e.g. cellular networks). Other landmark-based or map-based approaches have also been proposed which use ultrasonic, sonar, or laser range sensors $[2,6]$.

In fact, each of these approaches has its own disadvantages and estimation limits. With Odometer, the error due to wheel slips could not be eliminated and will keep adding up. Inertial navigating system (INS) is sensitive to vibration, noise and has temperature dependent measurement drifts. The accuracy range of global positioning system (GPS) is often several meters.

Thus, some hybrid positioning systems have been studied using two or more of the above technologies. The best example would be combinations of odometers, GPS, and INS sensors to locate vehicle position $[1,5,8]$. Based on the fact that INS provides high-rate position, velocity, and attitude data with good shortterm stability while the GPS provides position and velocity data with a good long-term stability [10], integrating the GPS with the INS has been largely used recently as a localization solution.

But with GPS and INS, the costs usually become quite expensive if we want to raise the precisions. Since autonomous vehicles are often equipped with a detection and ranging sensor to avoid collision, we propose a data merging algorithm using both laser detection and ranging (ladar) sensor and odometer data to plot out the environment map and to locate the vehicle position.

The paper is outlined as follows. In section 2, a simple bicycle mathematical model is given for the vehicle in order to design our localization algorithm. Then, in section 3 our algorithm is explained in detail. A Kalman filter which merge the sensor data and correct the final map plot is described. The algorithm is implemented and tested on a prototype electric car "CyCab" and the result is shown in section 4. Finally, we give a conclusion and discuss the future perspective of our research work in section 5 .

\section{Vehicle Model}

The mathematic model we use to develop the algorithm is a very simple planer model. We suppose that the vehicle behaves as a bicycle and that the wheels do not slip. The vehicle can be then drawn as shown in figure 1 .

From the above assumptions, we can write down the following equations in the vehicle's coordinate sys- 
tem:

$$
\left\{\begin{array}{l}
V_{1 x}=V_{2 x}=V_{o x} \\
V_{1 y}=V_{o y}+L_{1} \dot{\psi}=L \dot{\psi} \\
V_{2 y}=V_{o y}-L_{2} \dot{\psi}=0 \\
\dot{\psi}=\frac{V_{o x}}{L} \tan \alpha .
\end{array}\right.
$$

where $V_{1 x}, V_{2 x}$, and $V_{o x}$ are the longitudinal velocities of the front wheel, the rear wheel, and the center of gravity in the vehicle's coordinate system, $V_{1 y}, V_{2 y}$, and $V_{o y}$ are the lateral velocities of the front wheel, the rear wheel, and the center of gravity in the vehicle's coordinate system, $\psi$ is the yaw angle, $\alpha$ is the steering angle.

If $V=\left(V_{o x}, V_{o y}\right)^{\prime}$ denote the velocity in the vehicle's coordinate system and $V_{G}=\left(V_{G x}, V_{G y}\right)^{\prime}$ denote the velocity in the global coordinate system, we have the following relationship:

$$
V_{G}=\mathbf{R} V
$$

where $\mathbf{R}$ stands for the transformation matrix from the vehicle coordinate to the world coordinate.

$$
\mathbf{R}=\left[\begin{array}{cc}
\cos \psi & -\sin \psi \\
\sin \psi & \cos \psi
\end{array}\right]
$$

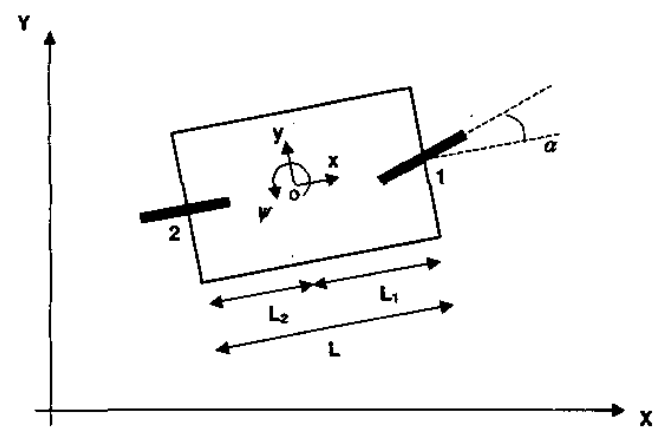

Figure 1: Bicycle model.

This very simple cinematic model will be used in the following section to determine in a first step the position and yaw angle of the vehicle. And then a Kalman filter based on the same model will be designed to correct the position on a map generated with the ladar data.

\section{Algorithm}

The goal of our research is to construit an algorithm that can plot out the map of the unknown environment in which the vehicle is operating, while at the same time, estimate and locate the vehicle's position on the generated map.

\subsection{General Philosophy}

We have chosen to use this stochastic map concept which was developed in the 80 's. This concept allows us to integrate data from the two different information sources we have (ladar and odometer) and to estimate statistically the vehicle states and the environment states at the same time by using a Kalman filter. Figure 2 shows the data flow and data processing diagram of our algorithm.

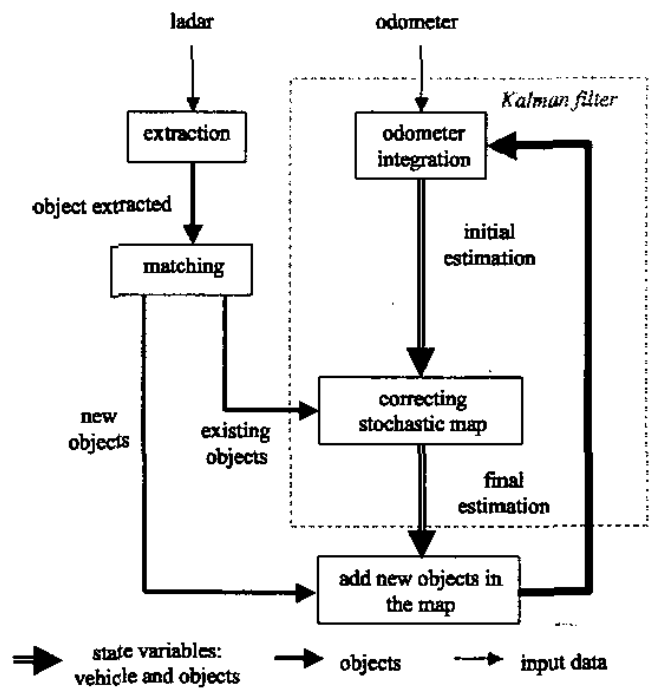

Figure 2: Algorithm process diagram.

First, the ladar scan plots out the operating environment map. An extracting algorithm locates objects in the operating environment and plot their positions to form a map. While the vehicle moves forward, the odometer integration algorithm estimates the vehicle position and also object positions in relation to the vehicle. Then, with a Kalman filter algorithm, we correct the object positions, thus the map, and also the vehicle positions on the map.

\subsection{System Equations for Extended Kalman Filter}

A Kalman filter is a recursive, linear, optimal, real time data processing algorithm which is used to estimate the states of a dynamic system in a noisy environment. It addresses the general problem of trying to estimate the state of a discrete-time controlled process that is governed by a linear stochastic difference equation. But since our system is non-linear, we try to linearize it about the current mean and covariance, and this is referred to as an extended Kalman filter [4].

From the equations (1) and (2), we can get the 
following discrete equations:

$$
\left\{\begin{array}{l}
x_{k}=x_{k-1}+\Delta l_{k} \cos \psi_{k-1} \\
y_{k}=y_{k-1}+\Delta l_{k} \sin \psi_{k-1} \\
\psi_{k}=\psi_{k-1}+\frac{\triangle l_{k}}{L} \tan \alpha_{k}
\end{array}\right.
$$

where $x_{k}, y_{k}$, and $\psi_{k}$ are the longitudinal, lateral position and yaw angle of the vehicle respectively in the world coordinate at $k^{\text {th }}$ time instant, $\Delta l_{k}=V_{o x k} \Delta t$ is the driving distance in $k^{t h}$ time interval $\Delta t$.

As the objects in the operating environment don't move, their positions can be expressed as follow:

$$
\begin{aligned}
& x_{G k}^{i}=x_{G k-1}^{i} \\
& y_{G k}^{i}=y_{G k-1}^{i} .
\end{aligned}
$$

$x_{G k}^{i}$ and $y_{G k}^{i}(i=1 \sim n)$ are the position of the $i^{t h}$ object in the world coordinate system.

So with equations (4) and (5), we can now form a discrete system to design our Kalman filter:

$$
\left\{\begin{array}{l}
X_{k}=F\left(X_{k-1}, \Delta l_{k}, \alpha_{k}\right)+v_{k-1} \\
Y_{k}=H\left(X_{k}\right)+w_{k}
\end{array}\right.
$$

with $v_{k}$ and $w_{k}$ the state variables noise and measurement noise respectively. The state variables $X_{k}$ and measurement output $Y_{k}$ at time instant $k$ are chosen as:

$$
X_{k}=\left[\begin{array}{c}
x_{k} \\
y_{k} \\
\psi_{k} \\
x_{G k}^{1} \\
y_{G k}^{1} \\
\vdots \\
x_{G k}^{i} \\
y_{G k}^{i} \\
\vdots \\
x_{G k}^{n} \\
y_{G k}^{n}
\end{array}\right], \quad Y_{k}=\left[\begin{array}{c}
x_{R k}^{1} \\
y_{R k}^{1} \\
\vdots \\
x_{R k}^{i} \\
y_{R k}^{i} \\
\vdots \\
x_{R k}^{n} \\
y_{R k}^{n}
\end{array}\right]
$$

The measurement output we get from the ladar are the object positions in the vehicle's coordinate system, which can be expressed as:

$$
\left[\begin{array}{c}
x_{R k}^{i} \\
y_{R k}^{i}
\end{array}\right]=H^{i}\left(X_{k}\right)=\mathbf{R}^{T}\left[\begin{array}{c}
x_{G k}^{i}-x_{k} \\
y_{G k}^{i}-y_{k}
\end{array}\right]+w_{k}^{i},
$$

for $i=1 \sim n$.

Thus, the linearized system equations of the system (6) can be written as:

$$
\left\{\begin{array}{l}
X_{k}=\mathbf{F}_{k-1} X_{k-1}+v_{k-1} \\
Y_{k}=\mathbf{H}_{k} X_{k}+w_{k}
\end{array}\right.
$$

with

$$
\mathbf{F}_{k}=\left.\frac{\partial F}{\partial X}\right|_{k}
$$

and

$$
\mathbf{H}_{k}=\left.\left(\frac{\partial H^{1}}{\partial X} \cdots \frac{\partial H^{i}}{\partial X} \cdots \frac{\partial H^{n}}{\partial X}\right)^{\prime}\right|_{k}
$$

\subsection{Extended Kalman filter}

The extended Kalman filter estimates a nonlinear system by using a form of feedback control: the filter estimates the system state variables at first and then obtains feedback in the form of measurements. Thus, the equations for the extended Kalman filter are divided into two groups: prediction equations and correction equations. It is shown in the following:

Prediction step:

$$
\begin{aligned}
& \hat{X}_{k}^{-}=F\left(\hat{X}_{k-1}, \Delta l_{k}, \alpha_{k}\right), \\
& \mathbf{P}_{k}^{-}=\mathbf{F}_{k} \mathbf{P}_{k-1} \mathbf{F}_{k}^{T}+v_{k} \mathbf{Q}_{k-1} v_{k}^{T} .
\end{aligned}
$$

Correction step:

$$
\begin{aligned}
\mathbf{K}_{k} & =\mathbf{P}_{k}^{-} \mathbf{H}_{k}^{T}\left(\mathbf{H}_{k} \mathbf{P}_{k}^{-} \mathbf{H}_{k}^{T}+w_{k} \mathbf{R}_{k} w_{k}^{T}\right)^{-1}, \\
\hat{X}_{k} & =\hat{X}_{k}^{-}+\mathbf{K}_{k}\left(Y_{k}-H\left(X_{k}^{-}\right)\right) \\
\mathbf{P}_{k} & =\left(\mathbf{I}-\mathbf{K}_{k} \mathbf{H}_{k}\right) \mathbf{P}_{k}^{-} .
\end{aligned}
$$

where $\mathbf{Q}$ is the state noise covariance, $\mathbf{R}$ is the measurement noise covariance, $\mathbf{P}$ is the error covariance, and $\mathbf{K}$ is the Kalman gain.

The prediction equations are responsible for projecting forward (in time) the current state and error covariance estimates to obtain the a priori estimates for the next time step (i.e. $\hat{X}_{k}^{-}$). The correction equations are responsible for the feedback for incorporating a new measurement into the a priori estimate to obtain an improved a posteriori estimate (i.e. $\hat{X}_{k}$ ).

\section{Experimental Result}

Our simultaneous map-plotting and localization algorithm was tested on a prototype electric car "CyCab" (see figure 3) designed especially for INRIA.

\subsection{CyCab}

The researchers of INRIA are working since 1991 on a new intelligent transportation system for the cities of tomorrow. We study in particular on two different concepts : car-sharing and the intelligent vehicle. The small electric vehicle named CyCab has been designed exactly to fulfill these two situations, especially for zones with limited access to regular automobiles. It can transport up to two persons in downtown areas, pedestrian malls, large industrial or amusement parks and airports, at a maximum of $30 \mathrm{~km} / \mathrm{h}$ speed. This revolutionary urban transportation vehicle is equipped with one computer to coordinate low 
level servo controls, one computer to realize high level HMI communication, a linear camera for platoon driving, a ladar sensor for anti-collision applications, a steering wheel angle encoder, and an odometer.

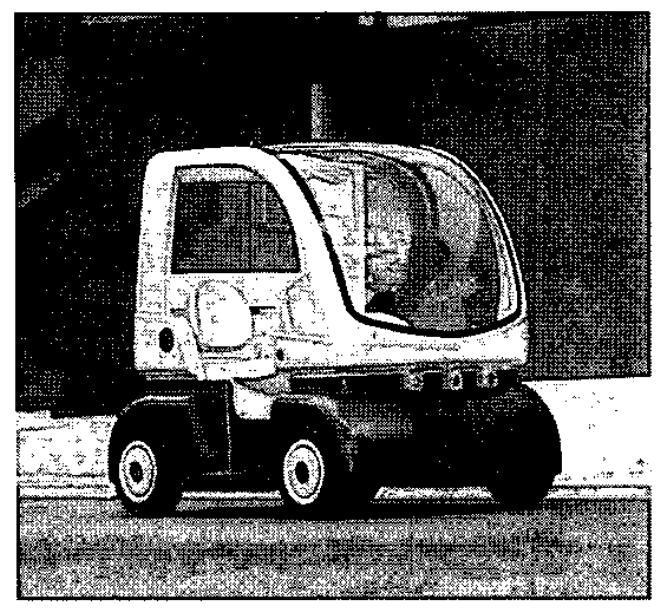

Figure 3: Autonomous urban vehicle "CyCab".

The ladar sensor (voir figure 4 ) is mounted to the front of our CyCab. It has a measurement range of at least $50 \mathrm{~m}$, with a distance resolution of $4 \mathrm{~mm}$ and accuracy of $5 \mathrm{~cm}$. The scan frequency is at $10 \mathrm{~Hz}$. It can give us a robust measurement of the objects in it's scanning range.

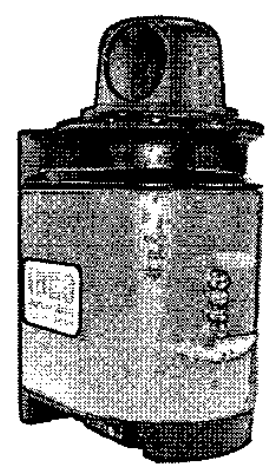

Figure 4: Laser detection and ranging sensor.

The encoder is installed in the differential gearbox located in the middle of the rear wheel axle. The resolution of the encoder is 100 pulses per revolution. It gives us the driving distance of the vehicle at a frequency of $100 \mathrm{~Hz}$. Together with the steering angle data, vehicle location can be estimated through the equation (4).
All the data transmission and servo control commande are managed by the low level servo control computer through CAN bus. These data can also be read and write by the high level HMI computer through the low level servo control computer.

\subsection{Experiment Setup}

First, we have placed some poles in the testing environment. The poles were of $90 \mathrm{~cm}$ tall, $5 \mathrm{~cm}$ in diameter, and were randomly placed. The reason of using poles to represent environment installation is because its simple geometry properties are easier to identify for a preliminary algorithm.

A driving test of a straight line was carried out in the testing environment using manual driving mode of $\mathrm{CyCab}$ with a driver on board. Our algorithm was launched on the high level HMI computer before the test. During the test, odometer and ladar sensor data were collected by the low level servo control computer, passed to the high level computer. Data were treated and environment map and vehicle position were plotted in real time by the high level computer on the screen on board as the CyCab was moving forward. The processing rate of our algorithm is at the frequency of the ladar sensor, which means the mapping and locating is updated every $100 \mathrm{~ms}$.

\subsection{Experimental Result}

The resulting map and driving trajectory was recorded and plotted as shown in figure 5. We can see that the cross points are the poles located by the ladar sensor and extracted with a preliminary recognition algorithm. Other environment obstacles are also drawn out with solid lines on the map.

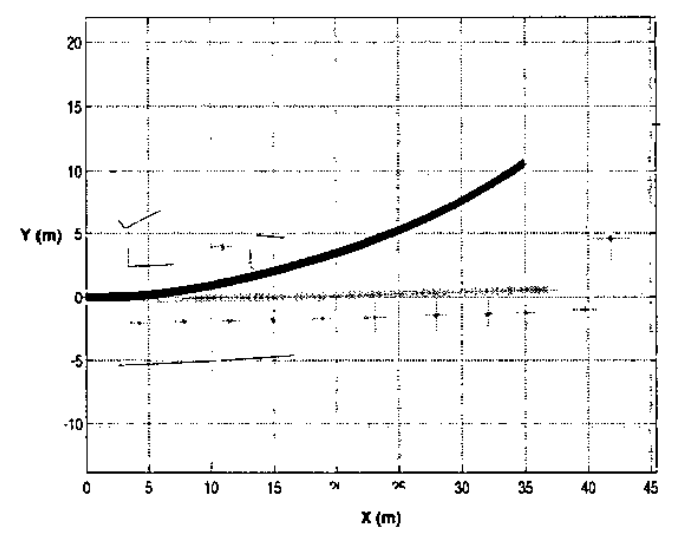

Figure 5: Experimental Result.

The black trajectory is traced out using only odometer data while the gray trajectory is the result of 
data merging and map correcting. With only odometer, the error can build up to $10 \mathrm{~m}$ in a test distance of only $35 \mathrm{~m}$, which is not acceptable at all. This is due to the measurement error on the steering angle and other measurement noise. These errors can build up throughout time when we estimate the vehicle position using equation (1).

On the other hand, the error is only a few centimeters with our data merging and map correcting algorithm using Kalman filtering technic.

\section{Conclusion and Future Perspective}

In this research work, a real time localization solution with a reasonable error and a small budget using ladar sensor and odometer is studied. A simultaneous map-plotting and localization algorithm is proposed using Kalman filter to merge collected data and to correct the resulting map plot. We show that our algorithm has improved significantly the position estimating result using only odometer data. Since autonomous vehicles are often equipped already with a detection and ranging sensor to avoid collision, the main advantage of our algorithm is that no extra sensors are needed in order to carry out the localization task.

The future perspective would be to integrate some cartographic data into the system and to use a mapmatching algorithm to give us the exact position for a localization task of larger scale. Further more, a crosswalk recognition algorithm by camera vision could also be included into the algorithm in order to perform larger scale repositioning. Finally, with a trajectory planing algorithm, we can realize a path following control algorithm so that the vehicle could be automatically navigated.

\section{References}

[1] I. Abuhadrous, F. Nashashibi, and C. Laurgeau, "3D Land Vehicle Localization: a Realtime Multi-sensor Data Fusion Approach Using RTMAPS," Proc. of the 11th International Conference on Advenced Robotics, Coimbra, Portugal, pp. 71-76, June 2003.

[2] J. Crowley, F. Wallner, and B. Schiele, "Position Estimation Using Principal Components of Range Data," Proc. of the 1998 IEEE International Conference on Robotics and Automation, Leuven, Belgium, pp. 3121-3128, May 1998.

[3] C. Drane and C. Rizos, Positioning Systems in Intelligent Transportation Systems, Artech House, 1998.

[4] M. Grewal and A. Andrews, Kalman Filtering: Theory and Practice Using MATLAB, J. Wiley \& Sons, 2001.
[5] M. Grewal, L. Weill, and A. Andrews, Global Positioning Systems, Inertial Navigation and Integration, J. Wiley \& Sons, 2nd edition, 2001.

[6] S. Lee, N. Amato, and J. Fellers, "Localization Based on Visibility Sectors Using Range Sensors," Proc. of the 2000 IEEE International Conference on Robotics and Automation, San Francisco, USA, pp. 3505-3511, April 2000.

[7] P. Laumond, La Robotique Mobile, HERMES Science Publications, Paris, 2001.

[8] S. Saab and Z. Kassas, "Map-Based Land Vihicle Navigation System with DGPS," Proc. of the 2002 IEEE Intelligent Vehicle Symposium, Versailles, France, pp. 209-214, June 2002.

[9] A. Victorino, La Commande référencée capteur: Une Approache Robuste au Problème de Navigation, Localization et Cartographie Simultanée pour un Robot d'Intérieur, $\mathrm{Ph}$. D. Thesis of Université de Nice-Sophia Antipolis, 2002.

[10] B. Vik and T. Fossen, "A Non-linear Observer for Integration of GPS and Inertial Navigation Systems," Modeling, Identification, and Control, Vol. 21, No. 4, October 2000.

[11] A. Walthelm, "A New Approach to Global Self-Localization with Laser Range Scans in Unstructed Environments," Proc. of the 2002 IEEE Intelligent Vehicle Symposium, Versailles, France, pp. 202-208, June 2002.

[12] J. Wang and C. Wilson, "Safety at the Wheel Improving KGPS/INS Performance and Reliability," GPS World, pp. 16-26, May 2003. 